\title{
Endoscopic Vacuum Therapy in Patients with Transmural Defects of the Upper Gastrointestinal Tract: A Systematic Review with Meta-analysis
}

\author{
Da Hyun Jung \\ Department of Internal Medicine, Severance Hospital, Yonsei University College of Medicine \\ Hae-Ryong Yun \\ Department of Internal Medicine, Yongin Severance Hospital, Yonsei University College of Medicine \\ Se Joon Lee \\ Department of Internal Medicine, Yongin Severance Hospital, Yonsei University College of Medicine \\ Na Won Kim \\ Medical Library, Yonsei University College of Medicine \\ Cheal Wung Huh ( $\sim$ huhcw@yuhs.ac) \\ Department of Internal Medicine, Yongin Severance Hospital, Yonsei University College of Medicine
}

\section{Research Article}

Keywords: Endoscopic vacuum therapy, aetiology, transmural defect, upper gastrointestinal tract

Posted Date: February 12th, 2021

DOI: https://doi.org/10.21203/rs.3.rs-152179/v1

License: (c) (i) This work is licensed under a Creative Commons Attribution 4.0 International License. Read Full License 


\section{Abstract}

A transmural defect of the upper gastrointestinal (UGI) tract is a life-threatening condition associated with high morbidity and mortality. Recently, endoscopic vacuum therapy (EVT) has shown rather excellent efficacy in managing UGI defects. We conducted a systematic review and meta-analysis to synthesise the available evidence on the efficacy of EVT in patients with transmural defects of the UGI tract. We searched the PubMed, Cochrane Library, and Embase databases for publications on the effect of EVT on successful closure, mortality, complications, and post-EVT stricture. Methodological quality was assessed using the Newcastle-Ottawa quality assessment scale. This meta-analysis included 29 studies involving 498 participants. The pooled estimate rate of EVT for successful closure was 0.85 ( $95 \%$ confidence interval [CI]: 0.81-0.88). The pooled estimate rate for mortality, complications, and post-EVT stricture was 0.11 , 0.10 , and 0.14 , respectively. According to the aetiology of the transmural defect (perforation vs. leak and fistula), no significant difference was found in successful closure (odds ratio [OR]: $1.45,95 \% \mathrm{Cl}: 0.45-4.67)$, mortality (OR: $0.77,95 \% \mathrm{Cl}: 0.24-2.46)$, complications (OR: $0.94,95 \% \mathrm{Cl}$ : $0.17-5.15)$, and postEVT stricture (OR: $0.70,95 \% \mathrm{Cl}: 0.12-4.24)$. The successful closure rate was significantly higher with EVT than with self-expanding metal stent (SEMS) placement (OR: 3.52, 95\% Cl: 1.79-6.91). In conclusion, EVT is an effective and safe treatment for treating leaks and fistulae as well as perforations in UGI defects. Moreover, EVT seems to be a better treatment option than SEMS placement in healing UGI defects.

\section{Background}

Transmural defects of the upper gastrointestinal (UGI) tract are categorised as perforations, leaks, or fistulae. A perforation is defined as an acute rupture of the gastrointestinal wall due to an endoscopic procedure, vomiting, foreign body, or peptic ulcer. A leak is a connection between the intraluminal and extraluminal spaces resulting from the disruption of the integrity of the wall, and the defect usually occurs after surgery. A fistula is defined as an abnormal communication between the gastrointestinal tract and other organs or the abscess cavity. Transmural defects of the UGI are life-threatening and associated with high morbidity and mortality.[1, 2] The optimal management of UGI transmural defects remains controversial. Surgery is one treatment strategy; however, the mortality rate has been known to be about $12-50 \% .[1,3,4]$ Placement of a self-expanding metal stent (SEMS) has also proven to be an effective treatment strategy for UGI defects.[5, 6] However, SEMS treatment could also cause complications such as stent migration, stent ingrowth, perforation, bleeding, epidural abscess, and vascular fistula.[7-9]

Recently, endoscopic vacuum therapy (EVT) has shown rather excellent efficacy in managing UGI defects.[10-12] This method applies continuous negative pressure to drain the infected fluid and accelerate wound healing.[13] EVT is suitable for localised defects for which stent placement is not possible. Moreover, an external drainage is not necessary in most cases.[14] However, the clinical success rate of EVT widely varies from 66.7-100\%.[15-17] In addition, corroborating evidence is needed because most studies are limited to case series and retrospective cohort studies with small sample sizes.

We performed a meta-analysis of the clinical outcomes of EVT in patients with transmural defects of the UGI tract. We aimed to assess the effect of EVT on successful closure, mortality, and postprocedural complications and stricture. In addition, we evaluated the efficacy of EVT according to the aetiology of the transmural defect (perforation vs. leak and fistula) and the treatment method (EVT vs. SEMS placement).

\section{Material And Methods}

\subsection{Literature search strategy}

We performed a systematic review and meta-analysis following the principles of the PRISMA (Preferred Reporting Items for Systematic reviews and MetaAnalyses) statement.[18] The PubMed, Cochrane Library, and Embase databases (from inception to April 2020) were independently searched by three authors (DHJ, HRY, and CWH). We used the following search string: anastomotic leak OR anastomotic leakage OR postoperative leak OR postoperative leakage OR oesophageal leak OR oesophageal leakage OR oesophageal fistula OR leakage OR fistula OR leak OR perforation OR upper gastrointestinal tract OR oesophagus OR oesophageal OR gastric OR stomach OR oesophagectomy OR anastomosis AND endoscopic vacuum therapy OR endoscopic vacuumassisted closure OR endoluminal vacuum therapy OR vacuum therapy OR vacuum-assisted closure OR negative pressure wound therapy OR endoscopic negative pressure therapy OR negative pressure therapy OR endovac therapy OR endo sponge. We manually and repetitively searched the cited references in published studies to identify other studies.

\subsection{Study selection}

In the first stage of the study selection, the title and abstract of articles that our keyword search returned were scrutinised to rule out irrelevant articles. Later, the full texts of all selected studies were screened according to our inclusion and exclusion criteria. The inclusion criteria were as follows: (1) a diagnosis of perforation, leak, or fistula of the UGI tract; (2) EVT as a primary or rescue treatment; and (3) investigations of adults aged $\geq 18$ years. The exclusion criteria were as follows: (1) article types other than original articles; (2) case reports including fewer than two patients; (3) abstract-only publications; and (4) publications in a language other than English. If several publications covering the same study population existed, only the most recent study was selected.

\subsection{Data extraction}

Three review authors ( $\mathrm{DHJ}, \mathrm{HRY}$, and $\mathrm{CWH}$ ) independently extracted data from the included studies using a pre-data extraction form. Further, we reviewed the titles and abstracts of all included studies to exclude irrelevant publications. Any discrepancies in data interpretation were resolved through a discussion, rereview of studies, and consultation with one other author (SJL). We extracted the following information: year of publication, first author, study design, patient age and sex, sample size, study region, follow-up duration, transmural defect size, time to diagnosis, time to treatment, EVT type, successful closure rate, mortality rate, complication rate, post-EVT stricture rate, hospital length of stay, intensive care unit length of stay, treatment duration, and number of sponge changes. 


\subsection{Primary and secondary outcomes}

The primary outcome was the successful closure rate. Successful closure was defined as no evidence of leak under direct endoscopic visualisation and the absence of contrast extravasation on either a computed tomography scan with oral contrast, an oesophagography, or a UGI study. The secondary outcomes were mortality rate, complication rate (Clavien-Dindo $\geq 3$ ), and stricture rate after EVT.

\subsection{Methodological quality}

The Newcastle-Ottawa quality assessment scale for cohort studies was used to evaluate the risk of bias. This scale rates studies on three sources of bias (selection, comparability, and outcome) based on eight criteria. Each criterion is rated with 1 star except comparability, which is rated a maximum of 2 stars. For this systematic review, studies scoring 7-9 stars were defined to be of high methodological quality, studies scoring 4-6 stars were defined to be of moderate methodological quality, and studies scoring 1-3 stars were defined to be of poor methodological quality. Three authors (CWH, HRY, and DHJ) independently evaluated the methodological quality of the selected studies. Any disagreement between the three authors was resolved by discussion.

\subsection{Statistical analysis}

A meta-analysis was performed using the statistical software R (version 3.3.3; R Foundation for Statistical Computing, Vienna, Austria). The Mantel-Haenszel random-effect model was applied for binary end points. The random-effect model was selected because it could consider the possibility of heterogeneity. In addition, we performed subgroup analyses according to the following criteria: closure rate, mortality rate, complication rate, post-EVT stricture rate according to the aetiology of the transmural defect (perforation vs. leak and fistula), closure rate of EVT and SEMS.

The $P$ test developed by Higgins was used for determining heterogeneity.[19] This test measures the percentage of total variation across studies. In cases of significant heterogeneity $(R>25 \%)$, the methodological section of each publication was re-evaluated for deciding whether any discrepancy could be checked. We used the Egger test to assess the extent of publication bias. A $p$ value of $<0.05$ was considered statistically significant.

\subsection{Institutional review board}

Approval by an internal review board was not applicable since only data which were already published was used for this study.

\section{Results}

\subsection{Study selection}

Overall, 2585 studies were identified. Duplicated articles $(n=392)$ were excluded. Further, 2144 articles were rejected based on the title and abstract. Forty-nine articles were fully reviewed. After the eligibility assessment, 20 articles were additionally excluded (Supplementary Fig. 1). A total of 29 articles were included involving 498 participants.[11, 12, 16, 20-45]

\subsection{Study characteristics and methodological quality}

The baseline characteristics of the included studies are listed in Table 1. Nineteen articles were retrospective cohort studies and 10 studies were case series studies. Eight studies included only patients with postoperative leak,[11, 21, 23, 24, 31, 40, 41, 44] and two studies included only patients with perforation.[22, 25] Eleven studies included patients with both postoperative leak and perforation.[12, 16, 27, 29, 30, 32, 33, 36, 38, 39, 42] Four studies included patients with fistula.[34, 35, 43, 45] Four studies compared EVT with SEMS.[20, 26, 28, 37] A total of 24 studies were conducted in Western countries (Germany 14, United States 4, Switzerland 2, United Kingdom 2, Portugal 1, and Australia 1), whereas 5 studies were conducted in Asia (Korea 4 and China 1). 
Table 1

Characteristics of the 29 studies included

\begin{tabular}{|c|c|c|c|c|c|c|c|c|c|c|c|}
\hline Authors & Study design & $\mathbf{N}$ & $\begin{array}{l}\text { Male } \\
\text { (n) }\end{array}$ & $\begin{array}{l}\text { Age } \\
\text { (median, } \\
\text { years) }\end{array}$ & $\begin{array}{l}\text { BMI } \\
\text { (median) }\end{array}$ & $\begin{array}{l}\text { Region of } \\
\text { study }\end{array}$ & $\begin{array}{l}\text { Follow- } \\
\text { up } \\
\text { (median, } \\
\text { months) }\end{array}$ & $\begin{array}{l}\text { Method of } \\
\text { diagnosis }\end{array}$ & $\begin{array}{l}\text { Defect } \\
\text { size } \\
\text { (median, } \\
\text { mm) }\end{array}$ & $\begin{array}{l}\text { Time to } \\
\text { diagnosis } \\
\text { (median, } \\
\text { days) }\end{array}$ & $\begin{array}{l}\text { T } \\
\text { tI } \\
(I \\
d\end{array}$ \\
\hline $\begin{array}{l}\text { Palmes } \\
2020\end{array}$ & Case series & Fistula: 4 & NA & NA & NA & $\begin{array}{l}\text { Western } \\
\text { (Germany) }\end{array}$ & MA & $\begin{array}{l}\text { Endoscopy } \\
\text { CT } \\
\text { Esophagogram }\end{array}$ & NA & NA & $\mathrm{N}$ \\
\hline Jung 2019 & Retrospective & $\begin{array}{l}\text { Leak: } 23 \\
\text { Perforation: } \\
7\end{array}$ & 20 & $65.1+$ & NA & $\begin{array}{l}\text { Western } \\
\text { (Germany) }\end{array}$ & $11.8+$ & NA & NA & $\begin{array}{l}\text { Leak: } 8.5 \\
\text { Perforation: } \\
<1\end{array}$ & $\mathrm{~N}$ \\
\hline Jeon 2019 & Retrospective & Leak: 22 & 17 & 68 & NA & $\begin{array}{l}\text { Eastern } \\
\text { (Korea) }\end{array}$ & 29.8 & $\begin{array}{l}\text { Endoscopy } \\
\text { CT } \\
\text { Esophagogram }\end{array}$ & NA & 11 & $\mathrm{~N}$ \\
\hline $\begin{array}{l}\text { Watson } \\
2019\end{array}$ & Case series & $\begin{array}{l}\text { Leak: } 2 \\
\text { Fistula: } 1\end{array}$ & 2 & $69.6 t$ & NA & $\begin{array}{l}\text { Western } \\
\text { (USA) }\end{array}$ & 2.7 & Endoscopy & NA & NA & $\mathrm{N}$ \\
\hline Pinto 2019 & Case series & Leak: 2 & 1 & $44.0+$ & NA & $\begin{array}{l}\text { Western } \\
\text { (Portugal) }\end{array}$ & NA & $\begin{array}{l}\text { Endoscopy } \\
\text { CT }\end{array}$ & NA & NA & $\mathrm{N}$ \\
\hline $\begin{array}{l}\text { Morell } \\
2019\end{array}$ & Case series & Leak: 6 & 2 & $49.0+$ & 44.2 & $\begin{array}{l}\text { Western } \\
\text { (Switzerland) }\end{array}$ & NA & $\begin{array}{l}\text { Endoscopy } \\
\text { СT }\end{array}$ & $20+$ & $4.5 t$ & 3 \\
\hline Min 2019 & Retrospective & Leak: 20 & 20 & 66.5 & NA & $\begin{array}{l}\text { Eastern } \\
\text { (Korea) }\end{array}$ & 7.1 & $\begin{array}{l}\text { Endoscopy } \\
\text { Esophagogram }\end{array}$ & 17.5 & 12.5 & 3 \\
\hline Loske 2019 & Case series & $\begin{array}{l}\text { Leak: } 1 \\
\text { Perforation: } \\
10\end{array}$ & 4 & $65.7+$ & NA & $\begin{array}{l}\text { Western } \\
\text { (Germany) }\end{array}$ & NA & $\begin{array}{l}\text { Endoscopy } \\
\text { CT }\end{array}$ & NA & NA & $\mathrm{N}$ \\
\hline Leeds 2019 & Retrospective & $\begin{array}{l}\text { Leak: } 54 \\
\text { Perforation: } \\
20\end{array}$ & NA & NA & NA & $\begin{array}{l}\text { Western } \\
\text { (USA) }\end{array}$ & NA & NA & NA & NA & $\mathrm{N}$ \\
\hline $\begin{array}{l}\text { Walsh } \\
2019\end{array}$ & Case series & $\begin{array}{l}\text { Leak: } 1 \\
\text { Perforation: } \\
1\end{array}$ & 1 & 69.5 & NA & $\begin{array}{l}\text { Western } \\
\text { (USA) }\end{array}$ & NA & NA & 20 & 21 & $\mathrm{~N}$ \\
\hline $\begin{array}{l}\text { Alakkari } \\
2019\end{array}$ & Case series & $\begin{array}{l}\text { Leak: } 1 \\
\text { Perforation: } \\
1\end{array}$ & 0 & $67+$ & NA & Western (UK) & NA & NA & NA & NA & 1 \\
\hline Berlth 2018 & Retrospective & $\begin{array}{l}\text { Leak: } 111 \\
\text { EVT: } 35 \\
\text { SEMS } 76\end{array}$ & 92 & $\begin{array}{l}\text { EVT: } 65 \\
\text { SEMS: } \\
64\end{array}$ & $\begin{array}{l}\text { EVT: } 26 \\
\text { SEMS: } \\
26\end{array}$ & $\begin{array}{l}\text { Western } \\
\text { (Germany) }\end{array}$ & NA & $\begin{array}{l}\text { Endoscopy } \\
\text { CT } \\
\text { Esophagogram }\end{array}$ & NA & $\begin{array}{l}\text { EVT: } 8 \\
\text { SEMS: } 8\end{array}$ & S \\
\hline Valli 2018 & Retrospective & $\begin{array}{l}\text { Leak: } 11 \\
\text { Fistula: } 1\end{array}$ & 9 & $\begin{array}{l}\text { Leak: } \\
57.5 \\
\text { Fistula: } \\
80\end{array}$ & NA & $\begin{array}{l}\text { Western } \\
\text { (Switzerland) }\end{array}$ & NA & $\begin{array}{l}\text { Endoscopy } \\
\text { СT } \\
\text { Esophagogram }\end{array}$ & NA & NA & $\begin{array}{l}\mathrm{L} \\
1 \\
\mathrm{~F} \\
5\end{array}$ \\
\hline Still 2018 & Retrospective & $\begin{array}{l}\text { Leak: } 2 \\
\text { Perforation: } \\
9 \\
\text { Fistula: } 2\end{array}$ & 6 & 63 & 23 & $\begin{array}{l}\text { Western } \\
\text { (USA) }\end{array}$ & NA & $\begin{array}{l}\text { Endoscopy } \\
\text { СT } \\
\text { Esophagogram }\end{array}$ & NA & NA & $\mathrm{N}$ \\
\hline $\begin{array}{l}\text { Pournaras } \\
2018\end{array}$ & Retrospective & $\begin{array}{l}\text { Leak: } 14 \\
\text { Perforation: } \\
7\end{array}$ & NA & NA & NA & Western (UK) & NA & NA & NA & NA & $\mathrm{N}$ \\
\hline
\end{tabular}




\begin{tabular}{|c|c|c|c|c|c|c|c|c|c|c|c|}
\hline Authors & Study design & $\mathbf{N}$ & $\begin{array}{l}\text { Male } \\
\text { (n) }\end{array}$ & $\begin{array}{l}\text { Age } \\
\text { (median, } \\
\text { years) }\end{array}$ & $\begin{array}{l}\text { BMI } \\
\text { (median) }\end{array}$ & $\begin{array}{l}\text { Region of } \\
\text { study }\end{array}$ & $\begin{array}{l}\text { Follow- } \\
\text { up } \\
\text { (median, } \\
\text { months) }\end{array}$ & $\begin{array}{l}\text { Method of } \\
\text { diagnosis }\end{array}$ & $\begin{array}{l}\text { Defect } \\
\text { size } \\
\text { (median, } \\
\text { mm) }\end{array}$ & $\begin{array}{l}\text { Time to } \\
\text { diagnosis } \\
\text { (median, } \\
\text { days) }\end{array}$ & $\begin{array}{l}\mathrm{T} \\
\mathrm{tI} \\
(\mathrm{I} \\
\mathrm{d}\end{array}$ \\
\hline Ooi 2018 & Retrospective & $\begin{array}{l}\text { Leak: } 6 \\
\text { Perforation: } \\
4\end{array}$ & NA & $56.7+$ & NA & $\begin{array}{l}\text { Western } \\
\text { (Australia) }\end{array}$ & NA & NA & 18.3 & NA & 3 \\
\hline Noh 2018 & Retrospective & Leak: 12 & 12 & 57.0 & NA & $\begin{array}{l}\text { Eastern } \\
\text { (Korea) }\end{array}$ & 12.9 & $\begin{array}{l}\text { CT } \\
\text { Esophagogram }\end{array}$ & 13 & 13.5 & 1 \\
\hline Loske 2018 & Case series & $\begin{array}{l}\text { Leak: } 3 \\
\text { Perforation: } \\
1\end{array}$ & NA & NA & NA & $\begin{array}{l}\text { Western } \\
\text { (Germany) }\end{array}$ & NA & NA & NA & NA & $\mathrm{N}$ \\
\hline $\begin{array}{l}\text { Laukoetter } \\
2017\end{array}$ & Retrospective & $\begin{array}{l}\text { Leak: } 39 \\
\text { Perforation: } \\
13\end{array}$ & 31 & 65 & NA & $\begin{array}{l}\text { Western } \\
\text { (Germany) }\end{array}$ & 5.4 & $\begin{array}{l}\text { Endoscopy } \\
\text { CT } \\
\text { Esophagogram }\end{array}$ & NA & NA & 8 \\
\hline $\begin{array}{l}\text { Kuehn } \\
2016\end{array}$ & Retrospective & $\begin{array}{l}\text { Leak: } 11 \\
\text { Perforation: } \\
10\end{array}$ & 15 & 72 & NA & $\begin{array}{l}\text { Western } \\
\text { (Germany) }\end{array}$ & 17 & $\begin{array}{l}\text { Endoscopy } \\
\text { CT } \\
\text { Esophagogram }\end{array}$ & NA & NA & $\mathrm{N}$ \\
\hline $\begin{array}{l}\text { Hwang } \\
2016\end{array}$ & Retrospective & $\begin{array}{l}\text { Leak: } 18 \\
\text { EVT: } 7 \\
\text { SEMS } 11\end{array}$ & 14 & $\begin{array}{l}\text { EVT: } \\
71.1 \\
\text { SEMS: } \\
67.3\end{array}$ & NA & $\begin{array}{l}\text { Eastern } \\
\text { (Korea) }\end{array}$ & NA & NA & $\begin{array}{l}\text { EVT: } 8.1 \\
\text { SEMS: } \\
6.6\end{array}$ & NA & $\mathrm{N}$ \\
\hline $\begin{array}{l}\text { Möschler } \\
2015\end{array}$ & Retrospective & $\begin{array}{l}\text { Leak: } 5 \\
\text { Perforation: } \\
5\end{array}$ & 5 & 73.9† & NA & $\begin{array}{l}\text { Western } \\
\text { (Germany) }\end{array}$ & 4 & NA & NA & NA & $\mathrm{N}$ \\
\hline $\begin{array}{l}\text { Mennigen } \\
2015\end{array}$ & Retrospective & $\begin{array}{l}\text { Leak: } 45 \\
\text { EVT: } 22 \\
\text { SEMS } 23\end{array}$ & 35 & $\begin{array}{l}\text { EVT: } 56 \\
\text { SEMS: } \\
65.5\end{array}$ & NA & $\begin{array}{l}\text { Western } \\
\text { (Germany) }\end{array}$ & $\begin{array}{l}\text { EVT: } 8.3 \\
\text { SEMS: } \\
16.8\end{array}$ & $\begin{array}{l}\text { Endoscopy } \\
\text { CT } \\
\text { Esophagogram }\end{array}$ & NA & $\begin{array}{l}\text { EVT: } 7 \\
\text { SEMS: } 7\end{array}$ & $\mathrm{~N}$ \\
\hline Loske 2015 & Case series & $\begin{array}{l}\text { Perforation: } \\
10\end{array}$ & NA & NA & NA & $\begin{array}{l}\text { Western } \\
\text { (Germany) }\end{array}$ & 2.8 & Endoscopy & 19 & $<1$ & $\mathrm{~N}$ \\
\hline Heits 2014 & Retrospective & $\begin{array}{l}\text { Perforation: } \\
10\end{array}$ & 5 & $66+$ & NA & $\begin{array}{l}\text { Western } \\
\text { (Germany) }\end{array}$ & 9 & $\begin{array}{l}\text { Endoscopy } \\
\text { CT } \\
\text { Esophagogram }\end{array}$ & 4.2 & NA & $\mathrm{N}$ \\
\hline Liu 2014 & Case series & Leak: 5 & NA & $61.8+$ & NA & $\begin{array}{l}\text { Eastern } \\
\text { (China) }\end{array}$ & NA & $\begin{array}{l}\text { Endoscopy } \\
\text { CT } \\
\text { Esophagogram }\end{array}$ & NA & 9.2 & N \\
\hline $\begin{array}{l}\text { Schorsch } \\
2013\end{array}$ & Retrospective & Leak: 17 & NA & NA & NA & $\begin{array}{l}\text { Western } \\
\text { (Germany) }\end{array}$ & NA & NA & 14.7 & 10 & $\mathrm{~N}$ \\
\hline $\begin{array}{l}\text { Schniewind } \\
2013\end{array}$ & Retrospective & Leak: 47 & NA & NA & NA & $\begin{array}{l}\text { Western } \\
\text { (Germany) }\end{array}$ & NA & NA & NA & NA & $\mathrm{N}$ \\
\hline $\begin{array}{l}\text { Brangewitz } \\
2013\end{array}$ & Retrospective & $\begin{array}{l}\text { EVT:32 } \\
\text { SEMS: } 39\end{array}$ & 58 & $\begin{array}{l}\text { EVT:63 } \\
\text { SEMS: } \\
62\end{array}$ & $\begin{array}{l}\text { EVT: } \\
25.2 \\
\\
\text { SEMS: } \\
26.4\end{array}$ & $\begin{array}{l}\text { Western } \\
\text { (Germany) }\end{array}$ & NA & NA & NA & NA & $\mathrm{N}$ \\
\hline
\end{tabular}

EVT, endoscopic vacuum therapy; CT, computed tomography; IC, intracavitary; IL, intraluminary; NA, not available; SEMS, self-expanding metal stent tData ex Table 2 summarises the clinical outcomes of the included studies. All studies except two [21, 34] reported the successful closure rate. Mortality was reported in all studies except one.[38] The complication and post-EVT stricture rates were reported in 21 and 16 studies, respectively. Hospital stay, intensive care unit stay, duration of therapy, and number of sponge changes were reported in 14, 5, 23, and 22 studies, respectively. 
Table 2

Clinical outcomes of the 29 studies included

\begin{tabular}{|c|c|c|c|c|c|c|c|c|c|}
\hline Authors & $\mathbf{N}$ & $\begin{array}{l}\text { Successful } \\
\text { closure rate (n, } \\
\% \text { ) }\end{array}$ & $\begin{array}{l}\text { Mortality } \\
\text { rate (n, } \\
\%)\end{array}$ & $\begin{array}{l}\text { Complication } \\
\text { rate (n, \%) }\end{array}$ & $\begin{array}{l}\text { Stricture } \\
\text { rate (n, } \\
\%)\end{array}$ & $\begin{array}{l}\text { Hospital stay } \\
\text { (median, } \\
\text { days) }\end{array}$ & $\begin{array}{l}\text { ICU stay } \\
\text { (median, } \\
\text { days) }\end{array}$ & $\begin{array}{l}\text { Duration of } \\
\text { therapy (median, } \\
\text { days) }\end{array}$ & $\begin{array}{l}\text { Sponge } \\
\text { changes }\end{array}$ \\
\hline $\begin{array}{l}\text { Palmes } \\
2020\end{array}$ & Fistula: 4 & $2 / 4(50)$ & $2 / 4(50)$ & NA & NA & NA & NA & 88.5 & NA \\
\hline \multirow[t]{2}{*}{ Jung 2019} & Leak: 23 & $20 / 23(87.0)$ & $\begin{array}{l}1 / 23 \\
(4.3)\end{array}$ & $0 / 23(0)$ & $\begin{array}{l}3 / 13 \\
(23.1)\end{array}$ & 54.4 & \multirow[t]{2}{*}{ NA } & 15.7 & 3.4 \\
\hline & $\begin{array}{l}\text { Perforation: } \\
7\end{array}$ & $5 / 7(71.4)$ & $\begin{array}{l}1 / 7 \\
(14.3)\end{array}$ & $0 / 7(0)$ & $\begin{array}{l}1 / 2 \\
(50.0)\end{array}$ & 33.7 & & 27.0 & 6.4 \\
\hline Jeon 2019 & Leak: 22 & 19/22 (86.4) & $0 / 22(0)$ & $0 / 22(0)$ & $\begin{array}{l}3 / 15 \\
(20.0)\end{array}$ & 24 & NA & 13 & 3 \\
\hline \multirow{2}{*}{$\begin{array}{l}\text { Watson } \\
2019\end{array}$} & Leak: 2 & $2 / 2(100.0)$ & $0 / 2(0)$ & $0 / 2(0)$ & $0 / 2(0)$ & \multirow[t]{2}{*}{ NA } & \multirow[t]{2}{*}{ NA } & 16 & 3 \\
\hline & Fistula: 1 & $1 / 1(100.0)$ & $0 / 1(0)$ & $0 / 1(0)$ & $0 / 1(0)$ & & & 40 & 9 \\
\hline Pinto 2019 & Leak: 2 & $1 / 2(50.0)$ & $0 / 2(0)$ & $0 / 2(0)$ & NA & NA & NA & 22.0 & 3 \\
\hline $\begin{array}{l}\text { Morell } \\
2019\end{array}$ & Leak: 6 & $6 / 6(100.0)$ & $0 / 6(0)$ & $0 / 6(0)$ & $0 / 6(0)$ & $39.8+$ & $10.2+$ & $32.3+$ & 4 \\
\hline Min 2019 & Leak: 20 & $19 / 20$ & $1 / 20$ & NA & $6 / 19$ & 49 & NA & 14.5 & 5 \\
\hline \multirow[t]{2}{*}{ Loske 2019} & Leak: 1 & $1 / 1(100.0)$ & $0 / 1(0)$ & \multirow[t]{2}{*}{ NA } & \multirow[t]{2}{*}{ NA } & \multirow[t]{2}{*}{ NA } & \multirow[t]{2}{*}{ NA } & \multirow[t]{2}{*}{11.0} & \multirow[t]{2}{*}{1.8} \\
\hline & $\begin{array}{l}\text { Perforation: } \\
10\end{array}$ & $10 / 10(100.0)$ & $\begin{array}{l}2 / 10 \\
(20.0)\end{array}$ & & & & & & \\
\hline \multirow[t]{2}{*}{ Leeds 2019} & Leak: 54 & $44 / 54(81.5)$ & \multirow[t]{2}{*}{ NA } & \multirow[t]{2}{*}{ NA } & \multirow[t]{2}{*}{ NA } & \multirow[t]{2}{*}{ NA } & \multirow[t]{2}{*}{ NA } & \multirow[t]{2}{*}{ NA } & \multirow[t]{2}{*}{ NA } \\
\hline & $\begin{array}{l}\text { Perforation: } \\
20\end{array}$ & $19 / 20(95.0)$ & & & & & & & \\
\hline \multirow[t]{2}{*}{$\begin{array}{l}\text { Walsh } \\
2019\end{array}$} & & \multirow[t]{2}{*}{$2 / 2(100.0)$} & \multirow{2}{*}{$\begin{array}{l}1 / 1 \\
(100.0)\end{array}$} & \multirow{2}{*}{$\begin{array}{l}0 / 1(0) \\
0 / 1(0)\end{array}$} & \multirow[t]{2}{*}{ NA } & NA & NA & 55 & 10 \\
\hline & $\begin{array}{l}\text { Perforation: } \\
1\end{array}$ & & & & & & & 42 & 3 \\
\hline Alakkari & Leak: 1 & $1 / 1(100.0)$ & $0 / 1(0)$ & $0 / 1(0)$ & $0 / 1(0)$ & NA & NA & 28 & 6 \\
\hline & $\begin{array}{l}\text { Perforation: } \\
1\end{array}$ & $1 / 1(100.0)$ & $0 / 1(0)$ & $0 / 1(0)$ & $0 / 1(0)$ & & & 56 & 13 \\
\hline Berlth 2018 & Leak: 111 & $30 / 35(85.7)$ & $4 / 35$ & $0 / 27(0)$ & $1 / 27$ & 39 & 8 & 12 & 3 \\
\hline & EVT: 35 & $55 / 76(72.4)$ & & $13 / 69(18.8)$ & & 37 & 7 & 28 & 1 \\
\hline & & & $\begin{array}{l}10 / 76 \\
(13.2)\end{array}$ & & $\begin{array}{l}5 / 69 \\
(7.2)\end{array}$ & & & & \\
\hline Valli 2018 & Leak: 11 & 9/11 (81.8) & $0 / 11(0)$ & $0 / 11(0)$ & $2 / 11$ & NA & NA & 20.8 & 5 \\
\hline & Fistula: 1 & $0 / 1(0)$ & $\begin{array}{l}1 / 1 \\
(100.0)\end{array}$ & $0 / 1(0)$ & $0 / 1(0)$ & & & 16 & 4 \\
\hline Still 2018 & Leak: 2 & NA & $1 / 13^{\ddagger}$ & $1 / 13^{\ddagger}$ & NA & NA & NA & NA & NA \\
\hline & $\begin{array}{l}\text { Perforation: } \\
9\end{array}$ & & & & & & & & \\
\hline & Fistula: 2 & & & & & & & & \\
\hline Pournaras & Leak: 14 & $14 / 14(100.0)$ & $0 / 14(0)$ & $1 / 14(7.1)$ & NA & 35 & NA & NA & 7 \\
\hline & $\begin{array}{l}\text { Perforation: } \\
7\end{array}$ & 6/7 (85.7) & $\begin{array}{l}1 / 7 \\
(14.3)\end{array}$ & $1 / 7(14.3)$ & & & & & \\
\hline Ooi 2018 & Leak: 6 & $4 / 6(66.7)$ & $1 / 6$ & 2/6 (33.3) & NA & 62 & 12 & $25.5 \dagger$ & $8.3+$ \\
\hline & $\begin{array}{l}\text { Perforation: } \\
4\end{array}$ & $2 / 4(50.0)$ & $\begin{array}{l}1 / 4 \\
(25.0)\end{array}$ & $1 / 4(25.0)$ & & & & & \\
\hline Noh 2018 & Leak: 12 & 10/12 (83.3) & $\begin{array}{l}1 / 12 \\
(8.3)\end{array}$ & $1 / 12(8.3)$ & $\begin{array}{l}1 / 12 \\
(8.3)\end{array}$ & NA & NA & 25 & $2.7+$ \\
\hline
\end{tabular}

EVT, endoscopic vacuum therapy; ICU, intensive care unit; NA, not available; SEMS, self-expanding metal stent tData expressed as mean. ${ }^{\ddagger}$ Only total rate was available. § Two patients died due to fatal hemorrhage during EVT. 


\begin{tabular}{|c|c|c|c|c|c|c|c|c|c|}
\hline Authors & $\mathrm{N}$ & $\begin{array}{l}\text { Successful } \\
\text { closure rate (n, } \\
\% \text { ) }\end{array}$ & $\begin{array}{l}\text { Mortality } \\
\text { rate (n, } \\
\%)\end{array}$ & $\begin{array}{l}\text { Complication } \\
\text { rate }(n, \%)\end{array}$ & $\begin{array}{l}\text { Stricture } \\
\text { rate }(n, \\
\%)\end{array}$ & $\begin{array}{l}\text { Hospital stay } \\
\text { (median, } \\
\text { days) }\end{array}$ & $\begin{array}{l}\text { ICU stay } \\
\text { (median, } \\
\text { days) }\end{array}$ & $\begin{array}{l}\text { Duration of } \\
\text { therapy (median, } \\
\text { days) }\end{array}$ & $\begin{array}{l}\text { Sponge } \\
\text { changes }\end{array}$ \\
\hline \multirow[t]{2}{*}{ Loske 2018} & Leak: 3 & 3/3 (100.0) & $0 / 3(0)$ & $0 / 3(0)$ & $0 / 3(0)$ & \multirow[t]{2}{*}{ NA } & \multirow[t]{2}{*}{ NA } & \multirow[t]{2}{*}{ NA } & \multirow[t]{2}{*}{ NA } \\
\hline & $\begin{array}{l}\text { Perforation: } \\
1\end{array}$ & $1 / 1(100.0)$ & $0 / 1(0)$ & $0 / 1(0)$ & $0 / 1(0)$ & & & & \\
\hline \multirow[t]{3}{*}{$\begin{array}{l}\text { Laukoetter } \\
2017\end{array}$} & \multirow[t]{2}{*}{ Leak: 39} & \multirow[t]{2}{*}{$36 / 39(92.3)$} & $\begin{array}{l}5 / 39 \\
(12.8)\end{array}$ & \multirow[t]{2}{*}{$2 / 39(5.1)^{\S}$} & $\begin{array}{l}4 / 39 \\
(10.2)\end{array}$ & \multirow[t]{2}{*}{60} & \multirow[t]{3}{*}{ NA } & \multirow[t]{2}{*}{20} & \multirow[t]{2}{*}{6} \\
\hline & & & 0/13 (0) & & $0 / 13(0)$ & & & & \\
\hline & $\begin{array}{l}\text { Perforation: } \\
13\end{array}$ & $13 / 13(100.0)$ & & 0/13 (0) & & 46 & & 24 & 6 \\
\hline \multirow{2}{*}{$\begin{array}{l}\text { Kuehn } \\
2016\end{array}$} & Leak: 11 & 9/11 (81.8) & $\begin{array}{l}1 / 11 \\
(18.2)\end{array}$ & \multirow[t]{2}{*}{ NA } & \multirow{2}{*}{$\begin{array}{l}1 / 11 \\
(18.2) \\
0 / 10(0)\end{array}$} & \multirow[t]{2}{*}{ NA } & \multirow[t]{2}{*}{ NA } & 12 & 4 \\
\hline & $\begin{array}{l}\text { Perforation: } \\
10\end{array}$ & $10 / 10(100.0)$ & $0 / 10(0)$ & & & & & 15 & 5 \\
\hline \multirow{3}{*}{$\begin{array}{l}\text { Hwang } \\
2016\end{array}$} & Leak: 18 & $7 / 7(100.0)$ & $0 / 7(0)$ & $0 / 7(0)$ & \multirow[t]{3}{*}{ NA } & 37.1 & \multirow[t]{3}{*}{ NA } & 19.5 & 4.3 \\
\hline & EVT: 7 & $7 / 11$ (63.6) & $\begin{array}{l}2 / 11 \\
(182)\end{array}$ & $6 / 11(54.5)$ & & 87.3 & & 27.0 & 1.6 \\
\hline & SEMS 11 & & & & & & & & \\
\hline \multirow{2}{*}{$\begin{array}{l}\text { Möschler } \\
2015\end{array}$} & Leak: 5 & $2 / 5(40.0)$ & \multirow{2}{*}{$\begin{array}{l}2 / 5 \\
(40.0) \\
0 / 5(0)\end{array}$} & $0 / 5(0)$ & \multirow{2}{*}{$\begin{array}{l}1 / 10 \\
(10.0)^{\S}\end{array}$} & \multirow[t]{2}{*}{38} & \multirow[t]{2}{*}{ NA } & 34.2 & 8.4 \\
\hline & $\begin{array}{l}\text { Perforation: } \\
5\end{array}$ & $5 / 5(100.0)$ & & $0 / 5(0)$ & & & & 13.0 & 2.0 \\
\hline \multirow{3}{*}{$\begin{array}{l}\text { Mennigen } \\
2015\end{array}$} & Leak: 45 & 19/22 (86.4) & \multirow[t]{2}{*}{$\begin{array}{l}3 / 22 \\
(13.6)\end{array}$} & 0/22 (0) & NA & 58 & NA & 26.5 & 6.5 \\
\hline & EVT: 22 & $14 / 23(60.9)$ & & $0 / 23(0)$ & & 53 & & 36.0 & 2.0 \\
\hline & SEMS 23 & & (26.1) & & & & & & \\
\hline Loske 2015 & $\begin{array}{l}\text { Perforation: } \\
10\end{array}$ & $10 / 10(100.0)$ & 0/10 (0) & 0/10 (0) & $0 / 10(0)$ & NA & NA & 5 & 2 \\
\hline Heits 2014 & $\begin{array}{l}\text { Perforation: } \\
10\end{array}$ & $9 / 10(90.0)$ & $\begin{array}{l}1 / 10 \\
(10.0)\end{array}$ & NA & NA & $48+$ & $22+$ & NA & $5.4 \dagger$ \\
\hline Liu 2014 & Leak: 5 & $5 / 5(100.0)$ & $0 / 5(0)$ & $0 / 5(0)$ & NA & NA & NA & 34.2 & NA \\
\hline $\begin{array}{l}\text { Schorsch } \\
2013\end{array}$ & Leak: 17 & $16 / 17(94.1)$ & $\begin{array}{l}1 / 17 \\
(5.9)\end{array}$ & NA & $\begin{array}{l}1 / 17 \\
(5.9)\end{array}$ & NA & NA & 12.0 & NA \\
\hline Schniewind & Leak: 29 & NA & $2 / 17$ & NA & NA & $57+$ & $26+$ & NA & NA \\
\hline & EVT: 17 & & (11.8) & & & $62+$ & $38+$ & & \\
\hline & SEMS 12 & & $\begin{array}{l}5 / 12 \\
(41.7)\end{array}$ & & & & & & \\
\hline $\begin{array}{l}\text { Brangewitz } \\
2013\end{array}$ & EVT:32 & $27 / 32(84.4)$ & $\begin{array}{l}5 / 32 \\
(156)\end{array}$ & $9 / 32(28.1)$ & $3 / 32$ & 48.5 & NA & 23 & 7 \\
\hline & SEMS: 39 & 21/39 (53.8) & (10.0) & $3 / 39$ (76.9) & $11 / 20$ & 41 & & 33 & 3 \\
\hline & & & $\begin{array}{l}11 / 39 \\
(28.2)\end{array}$ & & $\begin{array}{l}11 / 39 \\
(28.2)\end{array}$ & & & & \\
\hline
\end{tabular}

The methodological quality of the studies is shown in Supplementary Table 1 . The quality was poor in 15 of the included studies[22, 23, 25, 27, 30, 32, 34-36, $39-43,45]$ and moderate in 14 studies.[11, 12, 16, 20, 21, 24, 26, 28, 29, 31, 33, 37, 38, 44]

\subsection{Primary and secondary outcomes}

\subsubsection{Primary outcome - successful closure rate}

Twenty-seven studies reported data on successful closure for 456 patients. The pooled estimate rate for successful closure was 0.85 ( $95 \%$ confidence interval [Cl]: $0.81-0.88$, Fig. 1). No heterogeneity was found among the studies $(R=0 \%, p=0.68)$. No publication bias was detected by the Egger test $(p=0.33)$.

\subsubsection{Secondary outcomes - mortality, complication, and post-EVT stricture rates}


Data on mortality were reported in 28 studies that showed the information of 412 patients. The pooled estimate rate for mortality was 0.11 ( $95 \%$ Cl: $0.09-$ 0.15 , Fig. 2, A). No heterogeneity was found among these studies $\left(I^{2}=0 \%, p=0.96\right)$. No publication bias was detected by the Egger test $(p=0.38)$. Twenty-one studies reported data on complications for 304 patients. The pooled estimate rate for complications was 0.10 (95\% Cl: 0.06-0.15, Fig. 2, B). A low heterogeneity was found among the studies $(R=13.8 \%, p=0.28)$. Publication bias was detected by the Egger test $(p<0.05)$. Sixteen studies reported data on post-EVT stricture for 240 patients. The pooled estimate rate for post-EVT stricture was 0.14 (95\% Cl: 0.10-0.20, Fig. 2, C). No heterogeneity was found among these studies $(R=0 \%, p=0.45)$. No publication bias was detected by the Egger test $(p=0.06)$.

\subsection{Subgroup analysis}

\subsubsection{Perforation vs. leak and fistula - successful closure, mortality, complication, and post-EVT stricture rates}

Evaluation of the successful closure rate according to the aetiology of the transmural defect was performed in 11 studies. The pooled analysis showed that the successful closure rate was similar between the perforation and leak groups (odds ratio [OR]: 1.45, 95\% Cl: 0.45-4.67, p=0.53; Fig. 3, A). A low heterogeneity was detected among the studies $(P=24.1 \%, p=0.24)$. Data on mortality according to the aetiology of the transmural defect were available for 10 studies. The analysis revealed no significant difference between the two groups in mortality rate (OR: $0.77,95 \%$ Cl: $0.24-2.46, p=0.66$; Fig. 3, B), with no heterogeneity $(R=0 \%, p=0.58)$. Eight studies reported data on complications according to the aetiology of the transmural defect. The pooled analysis showed that the complication rate was similar between the perforation and leak groups (OR: $0.94,95 \% \mathrm{Cl}: 0.17-5.15, p=0.94 ;$ Fig. 3, C). No heterogeneity was detected among the studies $(R=0 \%, p=0.79)$. Data of post-EVT stricture rate according to the aetiology of the transmural defect were available for five studies. No significant difference between the two groups was observed in the post-EVT stricture rate (OR: 0.70, 95\% Cl: 0.12-4.24, $p=0.70 ;$ Fig. 3, D), with no heterogeneity $(R=0 \%, p=0.47)$.

\subsubsection{Successful closure rate (EVT vs. SEMS)}

The analysis of four studies involving 245 participants (96 in the EVT group and 149 in the SEMS group) showed a significant high successful closure rate in the EVT group (OR: 3.52, 95\% Cl: 1.79-6.91, $p<0.05$; Fig. 4), with no heterogeneity $(R=0 \%, p=0.75)$.

\section{Discussion}

To date, many studies have shown promising outcomes in patients with transmural defects of the UGI tract with EVT as a definitive treatment. However, those previous studies included only a limited number of patients. Recently, several systematic reviews were reported on the usefulness of EVT in transmural defects of the UGI tract.[17, 46-48] However, those previous reviews were only descriptive and did not show any statistical analysis with a summary estimate. Therefore, a meta-analysis was needed to synthesise the available data on the efficacy of EVT in transmural defects of the UGI tract. Our meta-analysis contained case series in which a single group was assessed with no within-study comparisons. Nevertheless, this meta-analysis has an advantage over a narrative review because it assessed effect sizes and integrated all of them in a single statistical analysis.

In this meta-analysis, the closure rate of EVT for transmural UGI defects was rather excellent (85\%), with low mortality (11\%), complication (10\%), and postEVT stricture (14\%) rates. And, no significant difference was found in successful closure (OR: 1.45, 95\% Cl: 0.45-4.67), mortality (OR: 0.77, 95\% Cl: 0.242.46), complications (OR: $0.94,95 \% \mathrm{Cl}: 0.17-5.15, p=0.94$ ), and post-EVT stricture (OR: $0.70,95 \% \mathrm{Cl}: 0.12-4.24, p=0.70)$ according to the aetiology of the transmural defect (perforation vs. leak and fistula). Although the aetiology of transmural UGI defects was different, the efficacy of EVT was similar between the groups. In addition, the successful closure rate was significantly higher with EVT than with SEMS (OR: 3.52, 95\% Cl: 1.79-6.91). To date, only one metaanalysis has reported studies comparing EVT and SEMS for the treatment of oesophageal defects.[49] In this previous study, the closure rate of oesophageal leak was also significantly higher with EVT than with SEMS, similar to our results. However, the number of included patients was smaller than that in our metaanalysis.

The principle of EVT is similar to the classical vacuum-assisted closure treatment, which is a well-established therapy for chronic superficial wounds. [50] In EVT, a polyurethane sponge is placed into the defect to treat it with the application of negative pressure. Defect healing is achieved through the continuous drainage of dirty fluid, thus decreasing bacterial colonisation, enhancing vascularity, and promoting tissue granulation.[50, 51] An internal vacuum sponge (endo-SPONGE) device was first successfully introduced for UGI anastomosis leak in 2008.[13] Since then, EVT has been used for the management of UGI defects and has shown good short-term and long-term clinical outcomes. Meanwhile, SEMS placement has also shown effective results for UGI defects.[5, 6] However, stent therapy may be accompanied by additional abscess drainage, local pressure necrosis of the mucosa, stent migration, stent ingrowth, bleeding, and perforation. Surgery is also one of the strategies for transmural defects of the UGl; however, it has been known to be associated with a high mortality rate. $[3,4]$ To date, comparative studies between different treatment modalities for UGI defects are rare.[52] Therefore, the clinical evidence on EVT for UGI defects is still insufficient for directing treatment modalities. According to our meta-analysis, EVT is an effective and safe treatment method for treating leaks and fistulae as well as perforations. In addition, EVT seems to have a significantly higher success rate in healing UGI defects than SEMS placement.

Usually, transmural defects of the UGI tract are classified as perforations, leaks, or fistulae. Of these, fistulae have been known to be the most difficult to close because the epithelial tract is often fibrotic and they arise in unhealthy tissue, which is inflamed, damaged, or ischaemic. Although the included cases were too 
few $(n=6)$, this meta-analysis showed a successful closure rate of $50 \%$ in patients with fistula. Given the poor response to other treatments such as SEMS placement, EVT would be promising option for treating patients with fistula.

The major disadvantages of EVT are the necessity for repetitive endoscopic procedures, nasogastric tube-related discomfort, and sponge dislocation. The main and most dreadful event associated with EVT is massive bleeding.[17, 46] It can occur from a fistula between the cavity and the main vessels and from rupture of a pseudoaneurysm from circumjacent vessels or heart chambers. More frequent changes of the sponge may be helpful to prevent or reduce the risk of severe bleeding. Moreover, massive bleeding can occur in cases of intracavitary therapy in which direct contact to blood vessels is possible. Therefore, intraluminary EVT may be safer than intracavitary EVT. Additionally, computed tomography scans should be reviewed before starting intracavitary EVT to exclude vascular issues. In our review, post-EVT stricture occurred in $14 \%$ cases. However, all strictures were easily resolved through endoscopic dilatations ( 26 cases).

\section{Limitations}

Although this is the first meta-analysis to assess the effect of EVT in patients with UGI defects, it had several limitations. All included studies were retrospective in nature without randomisation. This could have resulted in a selection bias in this study. Although randomised controlled trials are considered the best method for evaluating a treatment effect, performing such trials would be difficult owing to ethical concerns and methodological difficulty. Second, the included studies had a limited quality. Third, the sample size of each study was insufficient. Therefore, additional data would be needed to define the role of EVT in patients with UGI defects. Finally, most of the included studies were from Western countries, especially Germany. Large-scale studies from additional regions are required to validate the usefulness of EVT for the treatment of UGI defects. Despite these limitations, this meta-analysis, to our knowledge, showed the most comprehensive results on EVT for UGI defects thus far.

\section{Conclusions}

This meta-analysis found that EVT is an effective and safe treatment for leaks and fistulae as well as perforations in UGI defects. In addition, EVT seems to be a better treatment option in healing UGI defects than SEMS placement.

\section{Declarations}

\section{Conflict of Interest Disclosure:}

None.

\section{References}

1. Bufkin, B. L., Miller, J. I. Jr. \& Mansour, K. A. Esophageal perforation: emphasis on management. Ann Thorac Surg. 61, 1447-1451 discussion 1451 1442 (1996).

2. Altorjay, A., Kiss, J., Voros, A. \& Bohak, A. Nonoperative management of esophageal perforations. Is it justified? Ann Surg. 225, 415-421 (1997).

3. Brinster, C. J. et al. Evolving options in the management of esophageal perforation. Ann Thorac Surg. 77, 1475-1483 (2004).

4. Rohatgi, A., Papanikitas, J., Sutcliffe, R., Forshaw, M. \& Mason, R. The role of oesophageal diversion and exclusion in the management of oesophageal perforations. Int J Surg. 7, 142-144 (2009).

5. Kauer, W. K., Stein, H. J., Dittler, H. J. \& Siewert, J. R. Stent implantation as a treatment option in patients with thoracic anastomotic leaks after esophagectomy. Surgical endoscopy. 22, 50-53 (2008).

6. Huh, C. W. et al. Treatment of benign perforations and leaks of the esophagus: factors associated with success after stent placement. Surg Endosc. $\mathbf{3 2}$, 3646-3651 (2018).

7. Boulis, N. M., Armstrong, W. S., Chandler, W. F. \& Orringer, M. B. Epidural abscess: a delayed complication of esophageal stenting for benign stricture. Ann Thorac Surg. 68, 568-570 (1999).

8. Ho, H. S. \& Ong, H. S. A rare life-threatening complication of migrated nitinol self-expanding metallic stent (Ultraflex). Surg Endosc. 18:, 347 (2004).

9. Swinnen, J. et al. Self-expandable metal stents for the treatment of benign upper Gl leaks and perforations. Gastrointest Endosc. 73, 890-899 (2011).

10. Bludau, M. et al. Results of endoscopic vacuum-assisted closure device for treatment of upper GI leaks. Surg Endosc. 32, 1906-1914 (2018).

11. Min, Y. W. et al. Endoscopic vacuum therapy for postoperative esophageal leak. BMC surgery. 19, 37 (2019).

12. Jung, C. F. M. et al. (2020) Impact of Endoscopic Vacuum Therapy with Low Negative Pressure for Esophageal Perforations and Postoperative Anastomotic Esophageal Leaks. Digestion:1-11

13. Wedemeyer, J., Schneider, A., Manns, M. P. \& Jackobs, S. Endoscopic vacuum-assisted closure of upper intestinal anastomotic leaks. Gastrointest Endosc. 67, 708-711 (2008).

14. Loske, G., Schorsch, T. \& Muller, C. Endoscopic vacuum sponge therapy for esophageal defects. Surg Endosc. 24, 2531-2535 (2010).

15. Smallwood, N. R., Fleshman, J. W., Leeds, S. G. \& Burdick, J. S. The use of endoluminal vacuum (E-Vac) therapy in the management of upper gastrointestinal leaks and perforations. Surg Endosc. 30, 2473-2480 (2016).

16. Laukoetter, M. G. et al. Successful closure of defects in the upper gastrointestinal tract by endoscopic vacuum therapy (EVT): a prospective cohort study. Surgical endoscopy. 31, 2687-2696 (2017). 
17. Virgilio, E., Ceci, D. \& Cavallini, M. Surgical Endoscopic Vacuum-assisted Closure Therapy (EVAC) in Treating Anastomotic Leakages After Major Resective Surgery of Esophageal and Gastric Cancer. Anticancer research. 38, 5581-5587 (2018).

18. Liberati, A. et al. The PRISMA statement for reporting systematic reviews and meta-analyses of studies that evaluate health care interventions: explanation and elaboration. J Clin Epidemiol. 62, e1-34 (2009).

19. Higgins, J. P., Thompson, S. G., Deeks, J. J. \& Altman, D. G. Measuring inconsistency in meta-analyses. BMJ. 327, 557-560 (2003).

20. Brangewitz, M. et al. Endoscopic closure of esophageal intrathoracic leaks: stent versus endoscopic vacuum-assisted closure, a retrospective analysis. Endoscopy. 45, 433-438 (2013).

21. Schniewind, B. et al. Endoscopic endoluminal vacuum therapy is superior to other regimens in managing anastomotic leakage after esophagectomy: a comparative retrospective study. Surg Endosc. 27, 3883-3890 (2013).

22. Heits, N. et al. Endoscopic endoluminal vacuum therapy in esophageal perforation. Ann Thorac Surg. 97, 1029-1035 (2014).

23. Liu, Y. N. et al. Reliable management of post-esophagectomy anastomotic fistula with endoscopic trans-fistula negative pressure drainage. World J Surg Oncol. 12:, 240 (2014).

24. Schorsch, T., Muller, C. \& Loske, G. [Endoscopic vacuum therapy of perforations and anastomotic insufficiency of the esophagus]. Chirurg. 85, 1081-1093 (2014).

25. Loske, G., Schorsch, T., Dahm, C., Martens, E. \& Muller, C. latrogenic perforation of esophagus successfully treated with Endoscopic Vacuum Therapy (EVT). Endosc Int Open. 3:, E547-551 (2015).

26. Mennigen, R. et al. Comparison of Endoscopic Vacuum Therapy Versus Stent for Anastomotic Leak After Esophagectomy. J Gastrointest Surg. 19, 12291235 (2015)

27. Moschler, O., Nies, C. \& Mueller, M. K. Endoscopic vacuum therapy for esophageal perforations and leakages. Endosc Int Open. 3:, E554-558 (2015).

28. Hwang, J. J. et al. (2016) Comparison of Endoscopic Vacuum Therapy and Endoscopic Stent Implantation With Self-Expandable Metal Stent in Treating Postsurgical Gastroesophageal Leakage.Medicine (Baltimore)95:e3416

29. Kuehn, F. et al. Surgical Endoscopic Vacuum Therapy for Defects of the Upper Gastrointestinal Tract. J Gastrointest Surg. 20, 237-243 (2016).

30. Loske, G. et al. Open-pore film drainage (OFD): a new multipurpose tool for endoscopic negative pressure therapy (ENPT). Endosc Int Open. 6, E865-E871 (2018).

31. Noh, S. M. et al. (2018) Endoscopic Vacuum-Assisted Closure Therapy in Patients with Anastomotic Leakage after Esophagectomy: A Single-Center Experience.Gastroenterol Res Pract2018:1697968

32. Ooi, G. et al. Indications and efficacy of endoscopic vacuum-assisted closure therapy for upper gastrointestinal perforations. ANZ J Surg. 88:, E257-E263 (2018).

33. Pournaras, D. J. et al. Endoluminal Vacuum Therapy (E-Vac): A Treatment Option in Oesophagogastric Surgery. World J Surg. 42, 2507-2511 (2018).

34. Still, S., Mencio, M., Ontiveros, E., Burdick, J. \& Leeds, S. G. Primary and Rescue Endoluminal Vacuum Therapy in the Management of Esophageal Perforations and Leaks. Ann Thorac Cardiovasc Surg. 24, 173-179 (2018).

35. Valli, P. V. et al. Stent-over-sponge (SOS): a novel technique complementing endosponge therapy for foregut leaks and perforations. Endoscopy. 50, 148153 (2018).

36. Alakkari, A. et al. First UK experience of endoscopic vacuum therapy for the management of oesophageal perforations and postoperative leaks. Frontline Gastroenterol. 10, 200-203 (2019).

37. Berlth, F. et al. Self-Expanding Metal Stents Versus Endoscopic Vacuum Therapy in Anastomotic Leak Treatment After Oncologic Gastroesophageal Surgery. J Gastrointest Surg. 23, 67-75 (2019).

38. Leeds, S. G., Mencio, M., Ontiveros, E. \& Ward, M. A. Endoluminal Vacuum Therapy: How I Do It. J Gastrointest Surg. 23, 1037-1043 (2019).

39. Loske, G., Rucktaeschel, F., Schorsch, T., Moenkemueller, K. \& Mueller, C. T. Endoscopic negative pressure therapy (ENPT) for duodenal leakage - novel repair technique using open-pore film (OFD) and polyurethane-foam drainages (OPD). Endosc Int Open. 7:, E1424-E1431 (2019).

40. Morell, B., Murray, F., Vetter, D., Bueter, M. \& Gubler, C. Endoscopic vacuum therapy (EVT) for early infradiaphragmal leakage after bariatric surgeryoutcomes of six consecutive cases in a single institution. Langenbecks Arch Surg. 404, 115-121 (2019).

41. Rodrigues-Pinto, E., Morais, R., Vilas-Boas, F., Pereira, P. \& Macedo, G. Role of endoscopic vacuum therapy, internal drainage, and stents for postbariatric leaks. VideoGIE. 4, 481-485 (2019).

42. Walsh, L. T. et al. Successful treatment of large cavity esophageal disruptions with transluminal washout and endoscopic vacuum therapy: a report of two cases. Ther Adv Gastrointest Endosc. 12:, 2631774519860300 (2019).

43. Watson, A. \& Zuchelli, T. Repair of upper-GI fistulas and anastomotic leakage by the use of endoluminal vacuum-assisted closure. VideoGIE. 4, 40-44 (2019).

44. Jeon, J. H. et al. Endoscopic Vacuum Therapy in the Management of Postoperative Leakage After Esophagectomy. World J Surg. 44, 179-185 (2020).

45. Palmes, D. et al. (2020) Management of Nonmalignant Tracheo- and Bronchoesophageal Fistula after Esophagectomy. Thorac Cardiovasc Surg

46. Kuehn, F., Loske, G., Schiffmann, L., Gock, M. \& Klar, E. Endoscopic vacuum therapy for various defects of the upper gastrointestinal tract. Surg Endosc. 31, 3449-3458 (2017).

47. Verstegen, M. H. P. et al. Management of intrathoracic and cervical anastomotic leakage after esophagectomy for esophageal cancer: a systematic review. World J Emerg Surg. 14:, 17 (2019). 
48. Newton, N. J., Sharrock, A., Rickard, R. \& Mughal, M. Systematic review of the use of endo-luminal topical negative pressure in oesophageal leaks and perforations. Dis Esophagus. 30, 1-5 (2017).

49. Rausa, E. et al. (2018) Comparison of endoscopic vacuum therapy versus endoscopic stenting for esophageal leaks: systematic review and metaanalysis. Dis Esophagus 31

50. Argenta, L. C. \& Morykwas, M. J. Vacuum-assisted closure: a new method for wound control and treatment: clinical experience. Ann Plast Surg. 38, 563576 discussion 577 (1997).

51. Wedemeyer, J. et al. Management of major postsurgical gastroesophageal intrathoracic leaks with an endoscopic vacuum-assisted closure system. Gastrointest Endosc. 71, 382-386 (2010).

52. Schaheen, L., Blackmon, S. H. \& Nason, K. S. Optimal approach to the management of intrathoracic esophageal leak following esophagectomy: a systematic review. Am J Surg. 208, 536-543 (2014).

\section{Figures}

\section{Study}

Palmes, 2020

Jeon, 2020

Jung, 2019

Walsh, 2019

Watson, 2019

Pinto, 2019

Morell, 2019

Min, 2019

Loske, 2019

Leeds, 2019

Alakkari, 2019

Pournaras, 2018

Berlth, 2018

Valli, 2018

Ooi, 2018

Noh, 2018

Loske, 2018

Laukoetter, 2017

Kuehn, 2016

Hwang, 2016

Moschler, 2015

Mennigen, 2015

Loske, 2015

Heits, 2014

Liu, 2014

Schorsch, 2013

Brangewitz, 2013

Random effects model
Events Total

$\begin{array}{rr}2 & 4 \\ 19 & 22 \\ 25 & 30 \\ 2 & 2 \\ 3 & 3 \\ 1 & 2 \\ 6 & 6 \\ 19 & 20 \\ 11 & 11 \\ 63 & 74 \\ 2 & 2 \\ 20 & 21 \\ 30 & 35 \\ 9 & 12 \\ 6 & 10 \\ 10 & 12 \\ 4 & 4 \\ 49 & 52 \\ 19 & 21 \\ 7 & 7 \\ 7 & 10 \\ 19 & 22 \\ 10 & 10 \\ 9 & 10 \\ 5 & 5 \\ 16 & 17 \\ 27 & 32 \\ & \\ & 456 \\ & \end{array}$

Proportion $\quad 95 \%-\mathrm{Cl}$ Weight

$0.50[0.07 ; 0.93] \quad 2.0 \%$

$0.86[0.65 ; 0.97] \quad 5.2 \%$

$0.83[0.65 ; 0.94] \quad 8.4 \%$

$1.00[0.16 ; 1.00] \quad 0.8 \%$

$1.00[0.29 ; 1.00] \quad 0.9 \%$

$0.50[0.01 ; 0.99] \quad 1.0 \%$

$1.00[0.54 ; 1.00] \quad 0.9 \%$

$0.95[0.75 ; 1.00] \quad 1.9 \%$

$1.00[0.72 ; 1.00] \quad 1.0 \%$

$0.85[0.75 ; 0.92] 18.9 \%$

$1.00[0.16 ; 1.00] \quad 0.8 \%$

$0.95[0.76 ; 1.00] \quad 1.9 \%$

$0.86[0.70 ; 0.95] \quad 8.6 \%$

$0.75[0.43 ; 0.95] \quad 4.5 \%$

$0.60[0.26 ; 0.88] \quad 4.8 \%$

$0.83[0.52 ; 0.98] \quad 3.4 \%$

$1.00[0.40 ; 1.00] \quad 0.9 \%$

$0.94[0.84 ; 0.99] \quad 5.7 \%$

$0.90[0.70 ; 0.99] \quad 3.6 \%$

$1.00[0.59 ; 1.00] \quad 0.9 \%$

$0.70[0.35 ; 0.93] \quad 4.2 \%$

$0.86[0.65 ; 0.97] \quad 5.2 \%$

$1.00[0.69 ; 1.00] \quad 1.0 \%$

$0.90[0.55 ; 1.00] \quad 1.8 \%$

$1.00[0.48 ; 1.00] \quad 0.9 \%$

$0.94[0.71 ; 1.00] \quad 1.9 \%$

$0.84[0.67 ; 0.95] \quad 8.5 \%$

$0.85[0.81 ; 0.88] 100.0 \%$

Figure 1

Pooled estimate rate for successful closure in patients with transmural defects of the upper gastrointestinal tract 


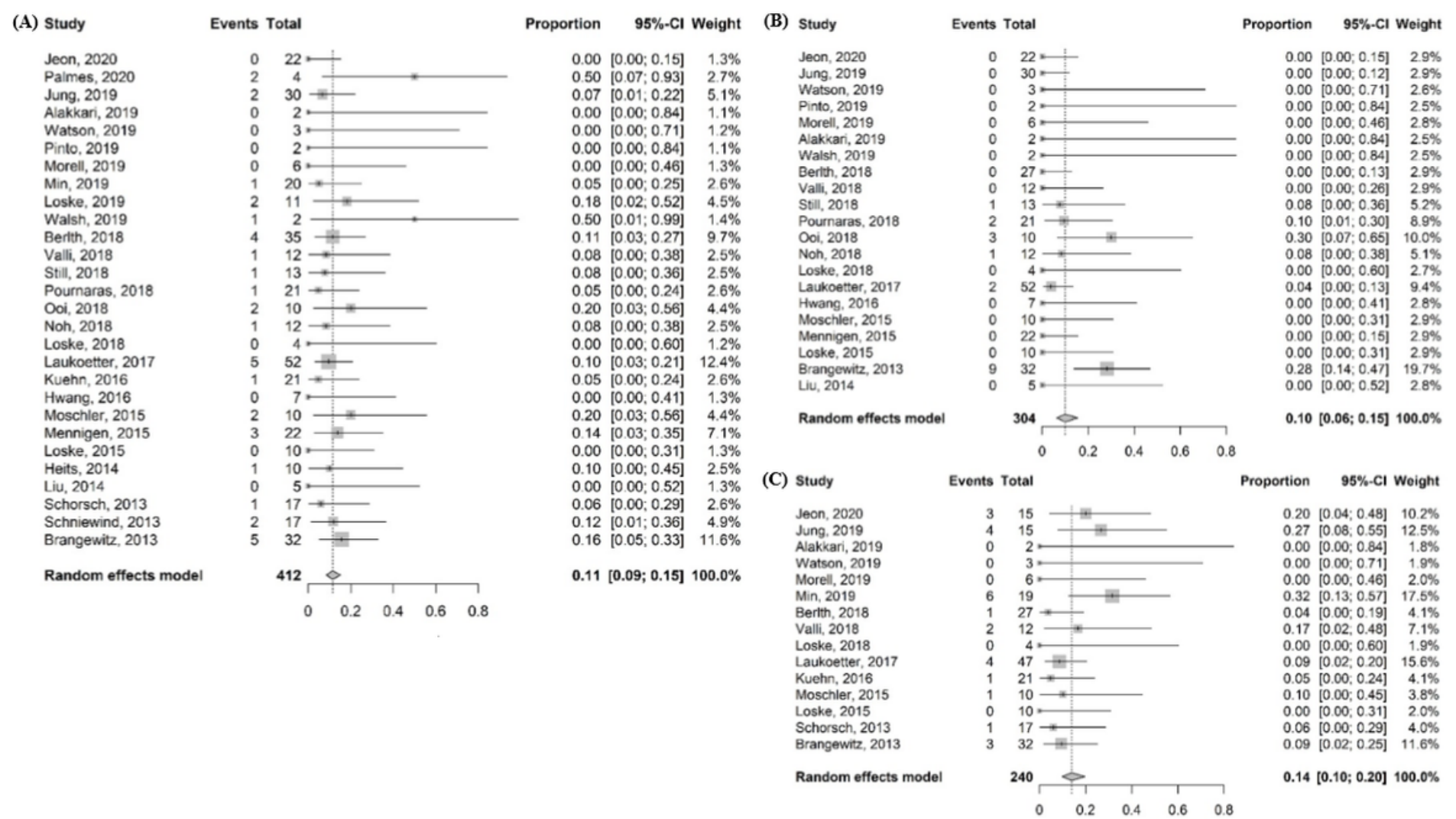

Figure 2

(A) Pooled estimate rate for mortality in patients with transmural defects of the upper gastrointestinal tract. (B) Pooled estimate rate for complications in patients with transmural defects of the upper gastrointestinal tract. (C) Pooled estimate rate for post-endoscopic vacuum therapy stricture in patients with transmural defects of the upper gastrointestinal tract.

(A)

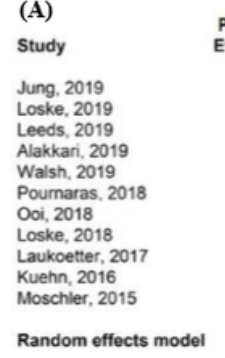

Perforation Leak \& fistula
Events Total Events Total

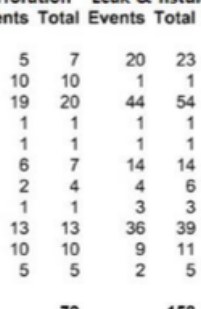

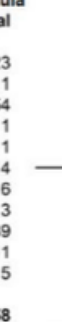

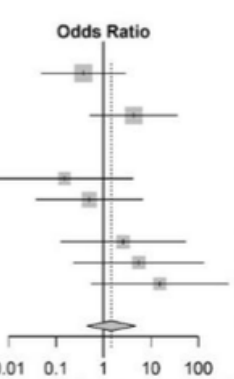

$\begin{array}{llll}\text { Favours [Leak] } & 1 & 10 & 100 \\ & \text { Favours [Perfo] } & & \end{array}$

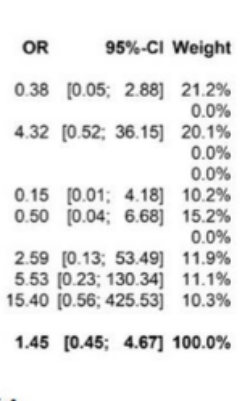

Study

Jung, 2019 Loske, 2019 Alakkari, 2019 Walsh, 2019 Pournaras, 2018 Ooi, 2018 Loske, 2018 Laukoetter, 2017 Kuehn, 2016 Moschier, 2015

Random effects model
Perforation Leak \& fistula Events Total Events Total

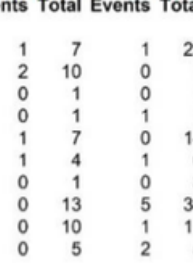

59

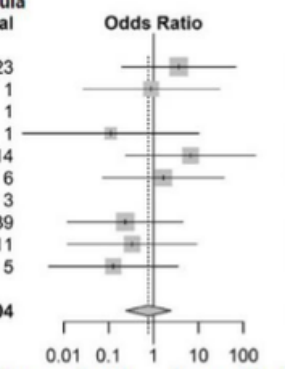

Favours [Leak] Favours [Perfo]
(C)

\section{Study}

Jung, 2019 Alakkari, 2019 Walsh, 2019 Pournaras, 201 Ooi, 2018 Loske, 2018 Moschier, 2015

Random effects model

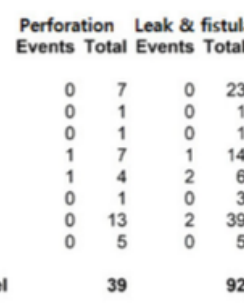

(D)

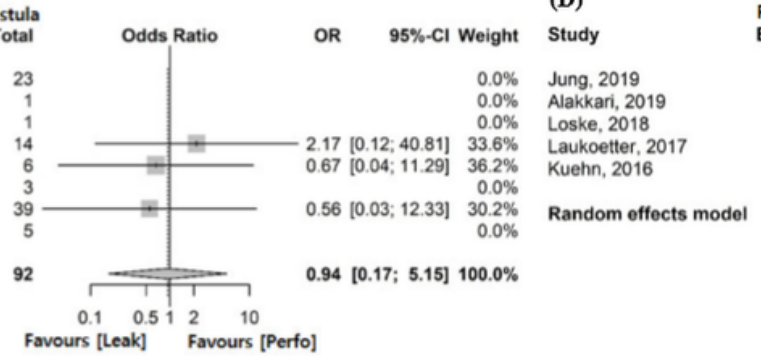

Perforation Leak \& fistula

Perforation Leak \& fistula

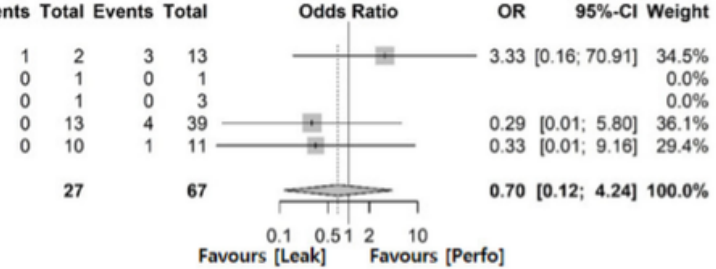
3.67 [0.20; 67.65] $16.0 \%$ 0.88 [0.03; 29.15] $11.1 \%$ $0.11[0.00 ; 10.27] \quad 6.6 \%$ $6.69[0.24 ; 187.28] \quad 12.2 \%$ $1.67[0.07 ; 37.73] \quad \begin{array}{r}13.9 \% \\ 0.0 \%\end{array}$ $0.23 \quad[0.01 ; 4.50] \quad 15.5 \%$ $\begin{array}{llll}0.33 & {[0.01 ;} & 9.16] & 12.4 \% \\ 0.13 & {[0.00 ;} & 3.52] & 12.3 \%\end{array}$ $0.77[0.24 ; 2.46] 100.0 \%$

\section{Figure 3}

(A) Forrest plot of successful closure rate for comparison between the perforation and leak groups. (B) Forrest plot of mortality rate for comparison between the perforation and leak groups. (C) Forrest plot of complication rate for comparison between the perforation and leak groups. (D) Forrest plot of postendoscopic vacuum therapy stricture rate for comparison between the perforation and leak groups. 


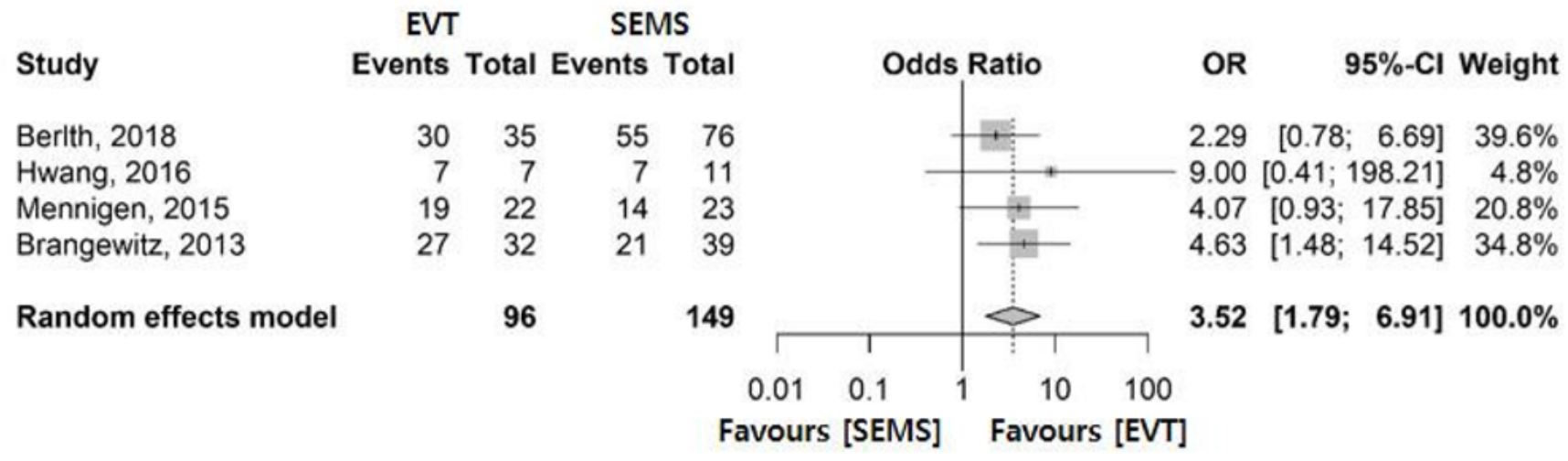

Figure 4

Forrest plot of successful closure rate for comparison between the endoscopic vacuum therapy and self-expanding metal stent groups

\section{Supplementary Files}

This is a list of supplementary files associated with this preprint. Click to download.

- Appendix.docx 